\title{
APPLICATION OF STEREOLOGICAL METHODS TO STUDY THE WHITE MATTER AND MYELINATED FIBERS THEREIN OF RAT BRAIN
}

\author{
SHU YANG ${ }^{1,2,3}$, CHEN LI $^{1,2,3}$, WEI ZHANG ${ }^{2,3}$, WEIWEI WANG ${ }^{3}$, JENS R NYENGAARD ${ }^{4}$ AND \\ YONG TANG ${ }^{2,3}$
}

${ }^{1}$ Department of Neurology, First Affiliated Hospital; ${ }^{2}$ Research Institute for Basic Medical Sciences; ${ }^{3}$ Department of Histology and Embryology, Chongqing Medical University, Chongqing, P. R. China; ${ }^{4}$ Stereology and Electron Microscopy Research Laboratory and MIND Center, Aarhus University, Aarhus, Denmark e-mail: Sheilayslamb@yahoo.com.cn; li.chen1107@yahoo.com.cn; zweihrui@yahoo.com.cn; wwwpzn@163.com; nyengaard@ki.au.dk; ytang062@yahoo.com.cn (Accepted March 23, 2008)

\begin{abstract}
An efficient and unbiased stereological method was applied to estimate the white matter volume, the total volume, total length and mean diameter of the myelinated fibers in the white matter and the total volume of the myelin sheaths in the white matter of rat brain. The white matter volume was obtained with the Cavalieri principle. Four tissue blocks were sampled from the entire white matter in a uniform random fashion. The length density of the myelinated fibers in the white matter was obtained from the isotropic, uniform, random sections ensured by the isector. The volume density of the myelinated fibers in the white matter was estimated by point counting. The total length and the total volume of the myelinated fibers in the white matter were estimated by multiplying the white matter volume and the length density and the volume density of the myelinated fibers in the white matter, respectively. The size of nerve fibers was derived by measuring the profile diameter perpendicular to its longest axis. The results were satisfactory in the sense that the sampling variance introduced by the stereological estimation procedure was a minor fraction of the observed variance. The comparison of the white matter and the myelinated fibers in the white matter between rat brain and human brain was also made in the present study.
\end{abstract}

Keywords: length, myelinated fibers, rat, stereology, volume, white matter.

\section{INTRODUCTION}

White matter changes in normal elderly human have been found by magnetic resonance imaging (MRI) (Christiansen et al., 1994; Ylikoski et al., 1995; Guttmann et al., 1998; Ge et al., 2002; Resnick et al., 2003) and by diffusion tensor imaging (DTI) (Malloy et al., 2007). However, MRI and DTI can not detect the microstructural changes of the nerve fibers in the white matter. Some previous studies have reported morphometric investigations of nerve fibers in the white matter during normal aging (Sturrock, 1976; 1987; Meier-Ruge et al., 1992; Sargon et al., 2003), but they did not use the design-based stereological techniques. Tang and Nyengaard (1997) described a stereological method for quantitatively studying the white matter and the myelinated fibers in the white matter of human brain. Human brain study could not completely exclude the brains with early stage of Alzheimer's disease. Since rats do not appear to develop Alzheimer's disease (Erickson and Barnes, 2003), they have become of increasing interest as a neuroscience research model. However, until now, the stereological methods have not been applied to investigate rat brain white matter and the myelinated fibers therein. Moreover, there has been no report comparing the white matter and the myelinated fibers in the white matter between human brain and rat brain. Therefore, the primary aims of the current study are to apply new stereological methods to measure the total length and total volume of the myelinated fibers in the rat white matter and to compare the current rat white matter data with previous human white matter data.

\section{MATERIALS AND METHODS}

Five young female (6-month old) Long-Evans rats were used. The rats were group-housed (2-3) in standard iron cages at a temperature of $22{ }^{\circ} \mathrm{C}$. They were kept under a constant 12-h light-dark cycle. Food and water could be obtained ad libitum. 


\section{ESTIMATION OF WHITE MATTER VOLUME}

The rats were first anaesthetized by $4 \%$ chloral hydrate intraperitoneally. Then, they were perfusionfixed with $2 \%$ paraformaldehyde and $2.5 \%$ glutaraldehyde in $0.4 \mathrm{M}$ phosphate buffered saline ( $\mathrm{pH} 7.4)$. After perfusion, the skull was dissected. Cerebellum, brain stem and cranial nerves attaching to pavimentum cerebri were cut off, and then the cerebral hemispheres were taken out. The two hemispheres were embedded in $6 \%$ agar. Each hemisphere was coronally cut into 2 mm-thick slabs, starting randomly at the frontal pole, with a mean number of slabs per hemisphere of 11 (range 10-12) (Fig. 1). A transparent counting grid with an area of $0.39 \mathrm{~mm}^{2}$ associated with each point was placed at random on the occipital surface of each slab. The points hitting the white matter were counted under anatomical microscope (Fig. 2). The total volume of white matter, V(wm), was calculated from Cavalieri's principle (Gundersen et al., 1988; Tang and Nyengaard, 1997; Tang et al., 1997; Marner et al., 2003; Tang et al., 2003; Tang and Nyengaard, 2004):

$$
\mathrm{V}(\mathrm{wm})=\mathrm{t} \times \mathrm{a}(\mathrm{p}) \times \sum \mathrm{P}(\mathrm{wm}),
$$

where $\mathrm{t}$ is the slab thickness, $\mathrm{a}(\mathrm{p})$ the area per point, and $\sum \mathrm{P}(\mathrm{wm})$ the total number of points hitting the white matter per rat.

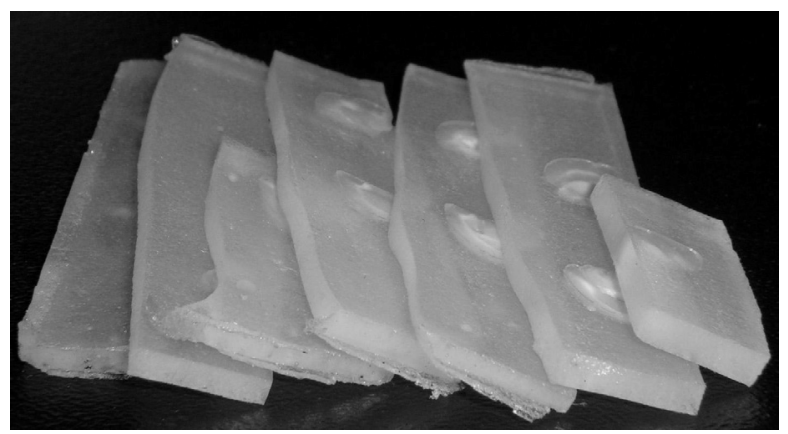

Fig. 1. 2 mm-thick consecutive slabs from a rat brain.

\section{SAMPLING OF WHITE MATTER}

Right or left hemisphere was sampled at random. From the slabs of the sampled hemisphere, every third slab was sampled systematically, the first one being chosen randomly. A plastic sheet with equidistant points was placed randomly on the occipital cut surface of the sampled slabs. Tissue blocks about 1 $\mathrm{mm}^{3}$ were sampled randomly from the entire white matter, including corpus callosum, internal capsule and anterior commissure, where the points in the plastic sheet hit the white matter (Fig. 3). Four tissue blocks were sampled per hemisphere. This sampling technique ensures a uniformly random distribution of the white matter samples. Consequently, the final samples represent all parts of the white matter rather than "ideal slices" chosen subjectively.

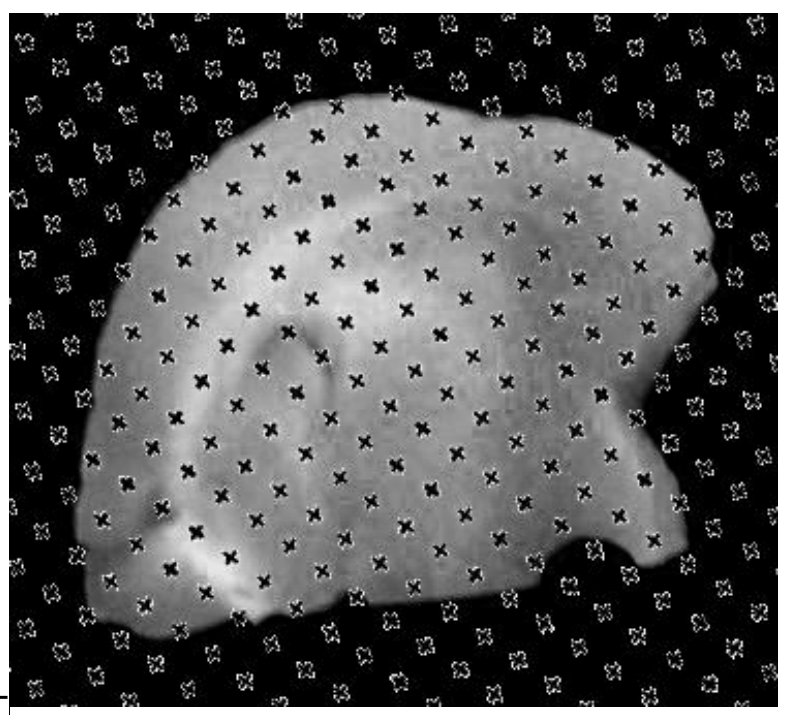

Fig. 2. A rat brain slice viewed under the microscope with an equidistant point grid superimposed.

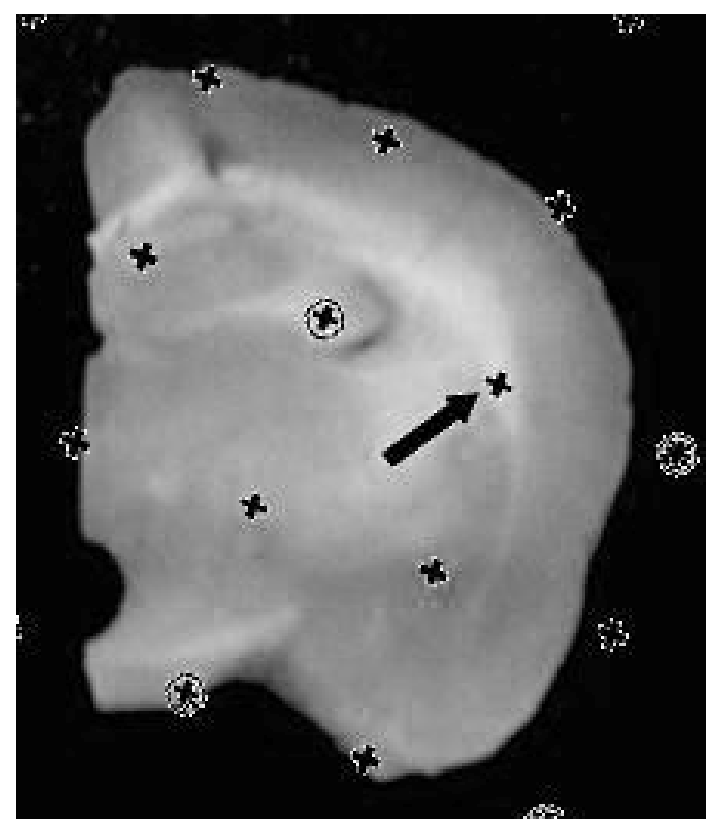

Fig. 3. An equidistant point grid randomly superimposed on a rat brain slice. The tissue block is sampled from white matter where the points in the plastic sheet hit the white matter, as indicated by arrow. 
The tissue blocks were fixed in 4\% glutaral-dehyde for at least $2 \mathrm{hrs}$ at $4{ }^{\circ} \mathrm{C}$ and rinsed in $0.1 \mathrm{M}$ phosphate buffered saline ( $\mathrm{pH}$ 7.2) 3 times, then osmicated in $1 \% 0.1 \mathrm{M}$ phosphate buffered osmium tetroxide $\left(\mathrm{OsO}_{4}\right)$ at $4{ }^{\circ} \mathrm{C}$ for $2 \mathrm{hrs}$. The blocks were gradually dehydrated through $50 \%, 70 \%$ and $90 \%$ ethanol followed by a mixture of $90 \%$ ethanol and $90 \%$ acetone, and finally $100 \%$ acetone. The blocks were then infiltrated with epoxy resin 618 (Chen Guang Chemical Industry, Sichuan, China). The infiltration steps were acetone: resin 1:1 ( $3 \mathrm{hrs}$ at room temperature), and absolute resin $\left(2 \mathrm{hrs}\right.$ at $\left.37^{\circ} \mathrm{C}\right)$. The tissue blocks were embedded in 5 $\mathrm{mm}$ epon spheres (Fig. 4), and the spheres were rotated randomly on the table before being re-embedded in oven at $37^{\circ} \mathrm{C}(16 \mathrm{hrs}), 45^{\circ} \mathrm{C}(12 \mathrm{hrs})$, and $60^{\circ} \mathrm{C}(14$ hrs). This method called isector makes sure that isotropic, uniform and random (IUR) sections can be obtained so that the fibers of each direction in 3dimensional space have the same probability to be sampled (Nyengaard and Gundersen, 1992).
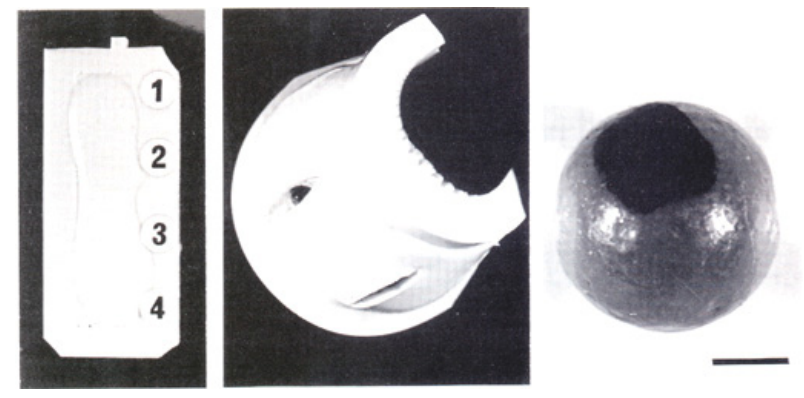

Fig. 4. Left: Isector moulds. The numbers on the mould indicate the position of $5 \mathrm{~mm}$ diameter spherical holes. Middle: A bent mould is illustrated. The holes receive the specimens and are filled with embedding medium. After hardening, the medium forms a sphere containing the specimen. Right: The specimen, previously stained black by uranyl acetate, is seen in a sphere of embedding medium.

One section with $60 \mathrm{~nm}$-thickness was cut from each epon block using a ultramicrotome. The ultrathin sections were then viewed in a transmission electron microscope (Hitachi-7500, made by Hitachi, Ltd., Japan) under operating voltage of $80 \mathrm{kV}$. Four fields of vision were randomly chosen and photographed at a TEM magnification of 6000 from each section.

\section{DENSITY ESTIMATES OF THE MYELINATED FIBERS AND MYELIN SHEATHS IN WHITE MATTER}

An unbiased counting frame (Gundersen, 1977), with an area of $3538 \mathrm{~mm}^{2}$ was overlaid randomly on the randomly captured photograph. The myelinated fiber profiles completely inside the counting frame or partly inside the counting frame but only touching the top and right lines were counted and the myelinated fiber profiles touching the bottom line, left line and its extension and the extension of right line were excluded for counting (Fig. 5). The length density of the myelinated fibers in white matter, $\operatorname{Lv}(\mathrm{nf} / \mathrm{wm})$, was estimated as (Gundersen et al., 1988; Tang and Nyengaard, 1997; Tang et al., 1997; Marner et al., 2003; Tang et al., 2003; Tang and Nyengaard, 2004):

$$
\mathrm{Lv}(\mathrm{nf} / \mathrm{wm})=2 \times \sum \mathrm{Q}(\mathrm{nf}) / \sum \mathrm{A}(\text { frame }),
$$

where $\sum \mathrm{Q}(\mathrm{nf})$ denotes the total number of the myelinated fiber profiles counted per rat white matter, $\sum \mathrm{A}$ (frame) the total area of counting frames used per rat.

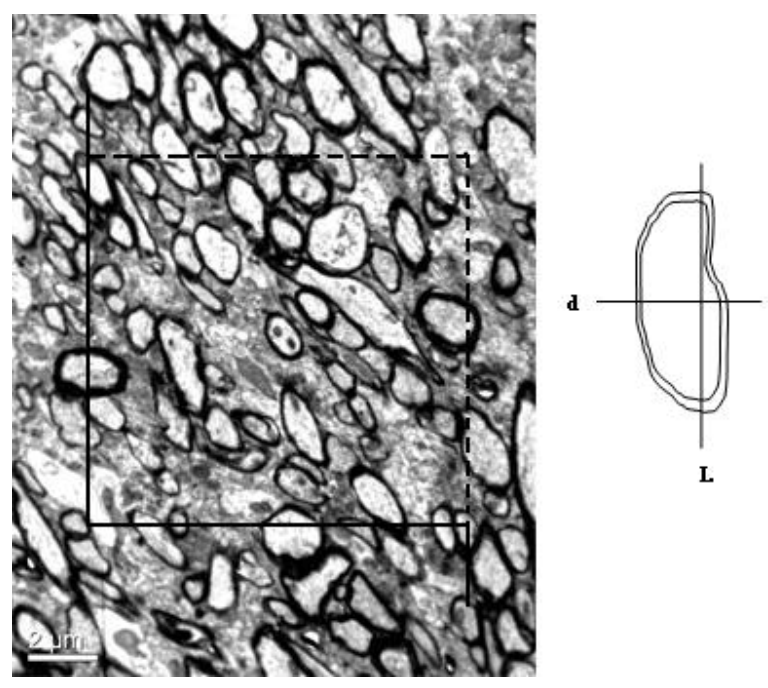

Fig. 5. Left: The rules of the unbiased counting frame: all nerve fiber profiles completely inside the counting frame or partly inside it but not touching or intersecting the full-drawn lines are considered for counting. Right: The diameter of the myelinated fibers (d) sampled with the unbiased counting frame is estimated by measuring the profile diameter perpendicular to its longest axis (L). 
A transparent counting grid with total number of points 396 was placed on the photograph. The points hitting the white matter, the myelinated fibers and the myelin sheaths surrounding fibers in the white matter were counted separately (Fig. 6). The volume density of the myelinated fibers in the white matter, $\mathrm{Vv}(\mathrm{nf} / \mathrm{wm})$, was estimated (see Gundersen et al., 1988; Tang and Nyengaard, 1997; Tang et al., 1997; Marner et al., 2003; Tang et al., 2003; Tang and Nyengaard, 2004):

$$
\mathrm{Vv}(\mathrm{nf} / \mathrm{wm})=\sum \mathrm{P}(\mathrm{nf}) / \sum \mathrm{P}(\mathrm{wm}),
$$

where $\sum \mathrm{P}(\mathrm{nf})$ is the total number of the points hitting the myelinated fibers in the white matter per rat, $\sum \mathrm{P}(\mathrm{wm})$ the total number of points hitting white matter per rat.

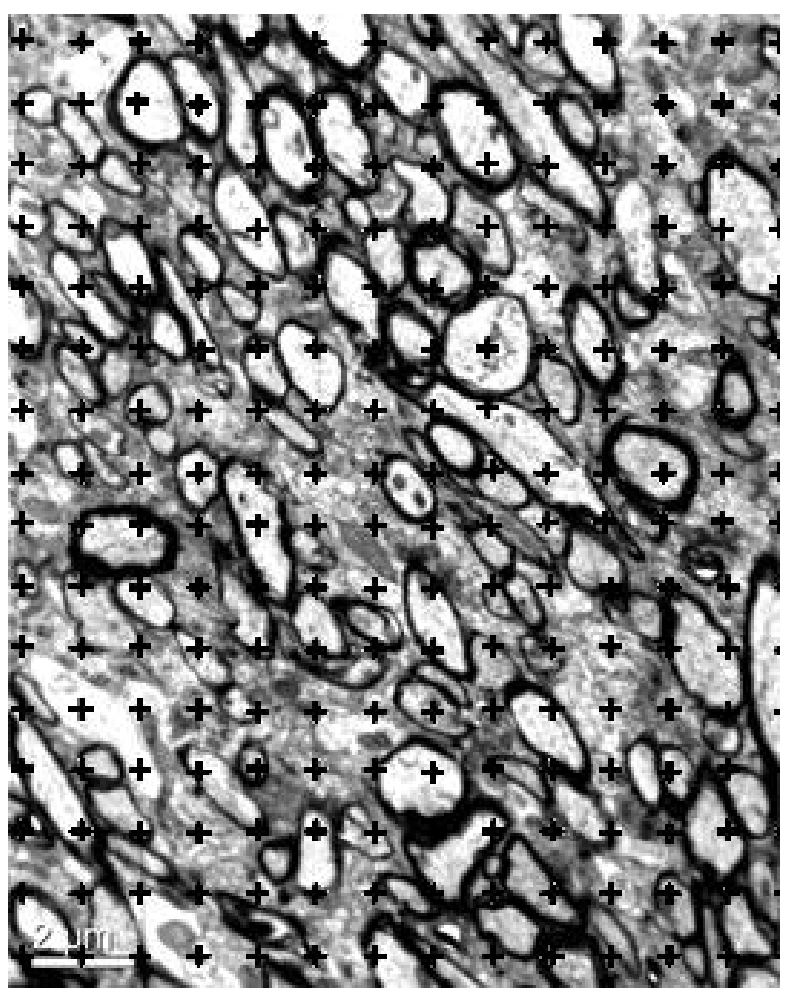

Fig. 6. The number of points hitting white matter, myelinated nerve fibers and myelin sheaths are counted separately.

The volume density of the myelin sheaths in the white matter, $\operatorname{Vv}(\mathrm{m} / \mathrm{wm})$, was estimated as (see Gundersen et al., 1988; Tang and Nyengaard, 1997; Tang et al., 1997; Marner et al., 2003; Tang et al., 2003; Tang and Nyengaard, 2004):

$$
\mathrm{Vv}(\mathrm{m} / \mathrm{wm})=\sum \mathrm{P}(\mathrm{m}) / \sum \mathrm{P}(\mathrm{wm}),
$$

where $\sum \mathrm{P}(\mathrm{m})$ is the total number of points hitting the myelin sheaths in the white matter per rat.

On average, 950 myelinated fiber profiles, 3300 points hitting the fibers and 1500 points hitting the myelin sheaths were counted per rat hemisphere.

\section{TOTAL QUANTITY ESTIMATES OF THE MYELINATED FIBERS AND MYELIN SHEATHS IN WHITE MATTER}

The total length and volume of mylinated fibers in the white matter and the total volume of their myelin sheaths were estimated by multiplying the respective component densities by white matter volume (Gundersen et al., 1988; Tang and Nyengaard, 1997; Tang et al., 1997; Marner et al., 2003; Tang et al., 2003; Tang and Nyengaard, 2004). For the total length of fibres, L(nf, wm):

$$
\mathrm{L}(\mathrm{nf}, \mathrm{wm})=\mathrm{Lv}(\mathrm{nf} / \mathrm{wm}) \times \mathrm{V}(\mathrm{wm}),
$$

for the total volume of fibres, $V(n f, w m)$ :

$$
\mathrm{V}(\mathrm{nf}, \mathrm{wm})=\mathrm{Vv}(\mathrm{nf} / \mathrm{wm}) \times \mathrm{V}(\mathrm{wm}),
$$

and, for the volume of myelin sheaths, $\mathrm{V}(\mathrm{m}, \mathrm{wm})$ :

$$
\mathrm{V}(\mathrm{m}, \mathrm{wm})=\mathrm{Vv}(\mathrm{m} / \mathrm{wm}) \times \mathrm{V}(\mathrm{wm}) .
$$

\section{ESTIMATION OF THE MEAN DIAMETER OF THE MYELINATED FIBERS IN THE WHITE MATTER}

With the assumption that nerve fibers are cylindrical tubes and the length of fibers is much longer than the thickness of sections plus the diameter of the fibers, the external diameter of the myelinated fibers was estimated by measuring the longest profile diameter perpendicular to its longest axis (Tang and Nyengaard, 1997; 2004) (Fig. 4).

\section{TISSUE SHRINKAGE}

We have previously determined the degree of white matter shrinkage with the same preparation as used in the present study (Tang and Nyengaard, 1997). The mean area shrinkage induced by this processing was $2.1 \%$, which was negligible. The area shrinkage in the Wistar rat for a similarly processed preparation to the current study was 1-6\% (Partadiredja et al., 2003). Therefore, the tissue shrinkage was not estimated in the present study.

\section{STATISTICS}

Variability within group was estimated using the dimensionless coefficient of variation $(\mathrm{CV}=\mathrm{SD} /$ Mean). The biological coefficient of variation $\left(\mathrm{CV}_{\text {bio }}\right)$ of the myelinated fiber volume and length among brains was estimated from the calculated stereological sampling variation (observed coefficient of error, OCE) and observed inter-brain coefficient of variation (OCV), using the relationship: $\mathrm{OCV}^{2}=\mathrm{CV}_{\text {bio }}{ }^{2}+\mathrm{OCE}^{2}$ (Kroustrup and Gundersen, 1983). The OCE was 
estimated from the estimated intra-brain coefficient of error using the relationship: $\mathrm{OCE}=\sqrt{\text { meanCE }}{ }^{2}$ (West et al., 1991). The CE of the Cavalieri estimator of the white matter volume, $\mathrm{CE}\left[\mathrm{V}_{(\mathrm{WM})}\right]$, in each brain was calculated according to Gundersen et al. (1999). $\mathrm{CE}\left[\mathrm{L}_{\mathrm{V}}(\mathrm{nf} / \mathrm{wm})\right]$ and $\mathrm{CE}\left[\mathrm{V}_{\mathrm{V}}(\mathrm{nf} / \mathrm{wm})\right]$ were calculated according to the following formula:

$$
C E_{n}\left(\frac{\Sigma Y}{\Sigma X}\right)=\sqrt{\frac{n}{n-1}\left(\frac{\Sigma(X)^{2}}{\Sigma X \Sigma X}+\frac{\Sigma(Y)^{2}}{\Sigma Y \Sigma Y}-\frac{2 \Sigma(X Y)}{\Sigma X \Sigma Y}\right)},
$$

where $\sum X$ is the number of the fields of vision used in each block when estimating the length density of the myelinated fibers or number of the fields of vision used in each block when estimating the volume density of the myelinated fibers. $\sum Y$ is the number of the myelinated fiber profiles in each block or number of the points hitting on the fiber profiles in each block and $\mathrm{n}$ is the number of blocks used when estimating the length or volume density of the myelinated fibers in the white matter.

The CE values for the estimates of the total volume and length of myelinated fibers in the white matter were calculated from the estimated coefficient of error for the corresponding component densities and the coefficient of error for white matter volume. The formulae are:

$$
\begin{aligned}
& C E[L(n f, w m)]=\sqrt{C E^{2}[L(n f / w m)]+C E^{2}[V(w m)]}, \\
& C E[V(n f, w m)]=\sqrt{C E^{2}[V(n f / w m)]+C E^{2}[V(w m)]} .
\end{aligned}
$$

\section{RESULTS}

The results from five rat brains are presented in Table 1 . The mean volume of the white matter is 120 (0.06) $\mathrm{mm}^{3}$ (CV in parenthesis), with a mean CE of 0.09 . The mean length density and the mean volume density of the myelinated fibers in the white matter are $1.08(0.21) \mathrm{km} / \mathrm{mm}^{3}$ and $0.49(0.15)$, respectively. The mean total length and the mean total volume of the myelinated fibers in the white matter are $129 \mathrm{~km}$ $(0.22)$ and $58.1(0.16) \mathrm{mm}^{3}$, respectively, with mean $\mathrm{CE}$ of 0.27 and 0.27 , respectively. The mean volume density and the mean total volume of the myelin sheaths in the white matter are $0.22(0.11)$ and 26.8 $(0.13) \mathrm{mm}^{3}$, respectively, with mean CE of 0.16 and 0.21 , respectively. The arithmetic mean diameter of the myelinated fibers is $0.56(0.05) \mu \mathrm{m}$, with a mean $\mathrm{CE}$ of 0.04 . The distribution of the length of the myelinated fibers in the white matter of 5 young female rats is shown in Fig. 7. The diameter of the myelinated fibers is mostly less than $1 \mu \mathrm{m}$. The average diameter of the myelinated fibers is $0.56 \mu \mathrm{m}$.

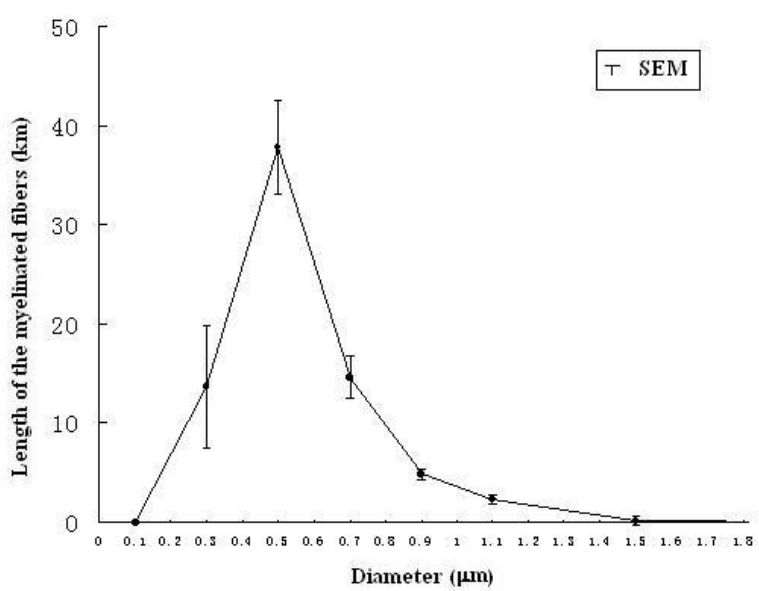

Fig. 7. The length of myelinated fibers with different diameters in the white matter is shown. The data has been pooled from 5 rats.

The comparison between the data of 5 young female rat brain white matter obtained from this present study and the data of 5 young female human brain white matter obtained from Tang and Nyengaard (1997)

\begin{tabular}{|c|c|c|c|c|c|c|c|c|c|c|c|c|c|c|c|c|}
\hline Animal & $\mathrm{V}(\mathrm{wm})$ & $\mathrm{CE}$ & $\mathrm{Vv}(\mathrm{NF})$ & $\mathrm{CE}$ & $\mathrm{V}(\mathrm{NF})$ & $\mathrm{CE}$ & Lv(NF) & $\mathrm{CE}$ & $\mathrm{L}(\mathrm{NF})$ & $\mathrm{CE}$ & $\mathrm{Vv}(\mathrm{ms})$ & $\mathrm{CE}$ & $\mathrm{V}(\mathrm{ms})$ & $\mathrm{CE}$ & $\mathrm{D}(\mathrm{NF})$ & $\mathrm{CE}$ \\
\hline & $\left(\mathrm{mm}^{3}\right)$ & $(\%)$ & & $(\%)$ & $\left(\mathrm{mm}^{3}\right)$ & $(\%)$ & $\mathrm{km} / \mathrm{mm}^{3}$ & $(\%)$ & $(\mathrm{km})$ & $(\%)$ & & $(\%)$ & $\left(\mathrm{mm}^{3}\right)$ & $(\%)$ & $(\mu \mathrm{m})$ & $(\%)$ \\
\hline 1 & 122 & 9.46 & 0.49 & 9.62 & $\begin{array}{l}59.8 \\
\end{array}$ & 13.5 & 0.73 & 18.3 & 88.8 & 20.6 & 0.25 & 6.05 & 30.2 & 11.2 & 0.59 & 5.20 \\
\hline 2 & 113 & 6.46 & 0.42 & 18.0 & 47.4 & 43.0 & 1.28 & 32.2 & 144 & 32.9 & 0.20 & 17.7 & 22.1 & 32.9 & 0.55 & 3.23 \\
\hline 3 & 122 & 10.7 & 0.41 & 24.2 & 50.1 & 26.5 & 1.06 & 13.4 & 130 & 17.1 & 0.20 & 15.5 & 24.3 & 18.9 & 0.57 & 3.42 \\
\hline 4 & 112 & 8.65 & 0.57 & 20.3 & 63.5 & 21.2 & 1.05 & 6.05 & 118 & 8.62 & 0.25 & 16.8 & 27.4 & 17.9 & 0.58 & 3.37 \\
\hline 5 & 129 & 7.91 & 0.54 & 20.8 & 70.0 & 22.2 & 1.28 & 40.6 & 166 & 41.4 & 0.23 & 18.7 & 30.2 & 20.3 & 0.51 & 3.27 \\
\hline Mean & 120 & 8.75 & 0.49 & 19.2 & 58.1 & 27.1 & 1.08 & 25.5 & 129 & 26.8 & 0.22 & 15.6 & 26.8 & 21.4 & 0.56 & 3.77 \\
\hline $\mathrm{CV}$ & 0.06 & & 0.15 & & 0.16 & & 0.21 & & 0.22 & & 0.11 & & 0.13 & & 0.05 & \\
\hline
\end{tabular}
as well as Tang et al. (2003) is presented in Table 2.

Table 1. Stereological results of the white matter and the myelinated fibers therein.

$\mathrm{V}(\mathrm{wm})$, the mean volume of the white matter; $\mathrm{Vv}(\mathrm{NF})$, volume density of the nerve fibers; $\mathrm{V}(\mathrm{NF})$, total volume of the nerve fibers; $\mathrm{Lv}(\mathrm{NF})$, length density of the nerve fibers; $\mathrm{L}(\mathrm{NF})$, total length of the nerve fibers; $\mathrm{Vv}(\mathrm{ms})$, volume density of the myelin sheaths; $\mathrm{V}(\mathrm{ms})$, total volume of the myelin sheaths; $\mathrm{D}(\mathrm{NF})$, arithmetic mean diameter of the nerve fiber. 
Table 2. The comparisons between young female human brain and young female rat brain.

\begin{tabular}{lcc}
\hline & Human brain $(38$-year on average) & Rat brain (6-month on average) \\
\hline $\mathrm{V}(\mathrm{wm})\left(\mathrm{mm}^{3}\right)$ & $475 \times 10^{3}$ & 120 \\
$\mathrm{~V}(\mathrm{wm})(\%)$ & 44.6 & 14.3 \\
$\mathrm{Vv}(\mathrm{NF})$ & 0.33 & 0.49 \\
$\mathrm{~V}(\mathrm{NF})\left(\mathrm{mm}^{3}\right)$ & $156 \times 10^{3}$ & 58.1 \\
$\mathrm{Lv}(\mathrm{NF})\left(\mathrm{km} / \mathrm{mm}^{3}\right)$ & 0.25 & 1.08 \\
$\mathrm{~L}(\mathrm{NF})(\mathrm{km})$ & $118 \times 10^{3}$ & 129 \\
$\mathrm{D}(\mathrm{NF})(\mu \mathrm{m})$ & 1.14 & 0.56 \\
\hline
\end{tabular}

$\mathrm{V}(\mathrm{wm})$, the mean volume of the white matter; $\mathrm{Vv}(\mathrm{NF})$, volume density of the nerve fibers; $\mathrm{V}(\mathrm{NF})$, total volume of the nerve fibers; $\mathrm{Lv}(\mathrm{NF})$, length density of the nerve fibers; $\mathrm{L}(\mathrm{NF})$, total length of the nerve fibers; $\mathrm{D}(\mathrm{NF})$, arithmetic mean diameter of the nerve fiber

\section{DISCUSSION}

This study for the first time combined transmission electron microscopy technique and unbiased stereological principle to quantitatively investigate the myelinated nerve fibers in the white matter of LongEvans rats.

In previous studies, only density of nerve fibers in the white matter has been obtained from 2-dimensional microscopic images (Sturrock, 1987; Meier-Ruge et al., 1992). When only reporting the density estimates, the possible age-related change in the reference space, i.e. the total volume of the white matter, was not taken into account. Biological conclusion drawn from density measurements was difficult to interpret since it would never be known whether any changes in the density were caused by an alteration of the total quantity and/or an alteration in the reference volume (Braendgaard and Gundersen, 1986). It was possible that the density was increased due to the shrinkage of the reference volume even though the total quantity was actually decreased. The present stereological method was designed to provide an estimate of the total quantities. The total length and total volume of the myelinated fibers and the total volume of the myelin sheaths in the white matter were obtained by multiplying the density estimates by the white matter volume. The white matter volume was obtained with the Cavalieri principle. Therefore, in the present study, the problem associated with density estimate was solved by estimating the total quantities with the design-based stereological method.

Besides the problem associated with density estimate, another methodological problem in previous studies in the white matter was that they did not sample the white matter uniformly (Sturrock, 1987; Meier-Ruge et al., 1992). They selected typical parts of a brain region of interest. The conclusions that were drawn from the analysis of standard sections or "ideal fields" in which the objects of interest were best identified could only apply to that part of tissue. The standard section or "ideal fields" were not representative of the entire tissue and thus introduced a bias. A uniform random sampling of the white matter was used in this study so that all parts of the white matter had an equal probability of being sampled. Since this study aims to get the information of the entire white matter, we uniformly and randomly sampled the tissues from the entire white matter. The present study did not take the variations between white matter regions into account. If the variations between the white matter regions need to be obtained, the tissues should be randomly sampled from each white matter region (Partadiredja et al., 2003). In addition, the presented data are from one hemisphere of rat brain by randomly sampling the right or left hemisphere. Therefore, this study does not consider side differences.

The myelinated fibers in the white matter are arranged irregularly, which complicates the precise quantitation. Uniform, 3-dimensional sampling strategy should be used to provide practically unbiased estimations. When estimating the length of fibers on 2-dimensional section, there is the rather strong requirement that either the fiber profiles are estimated from isotropic, uniform and random sections or the fiber profiles should be isotropic. In this study, isotropic, uniform random (IUR) sections were ensured by the isector so that all myelinated fibers in 3-dimensions had an equal probability of being estimated (Nyengaard and Gundersen, 1992). The results could represent the entire myelinated fibers in the white matter. Moreover, in this study, an unbiased counting frame (Gundersen, 1977; Tang and Nyengaard, 1997; Tang et al. 1997, Partadiredja et al. 2003) was used to ensure that the two-dimensional counting was correct.

In this study, we cut very thin sections $(60 \mathrm{~nm})$ to avoid the overprojection bias caused by the section thickness (Gundersen, 1979). The estimation of the length density and volume density of the myelinated fibers in the white matter demands the identification 
of the profiles of myelinated fibers. We used transmission electron microscopy to detect the myelinated fibers under a magnification of 6000 . Four fields of vision were randomly chosen and photographed from each section. In total, there were 16 photographs for a rat. The very thin sections and the use of the higher resolution of TEM ensured the result's accuracy.

A series of behavioral tests have reflected that many behaviors of rat are more or less related to those of human (Pang et al., 1992; Shu et al., 2002; Wallace et al., 2006; de Wit et al., 2007; Lammers et al., 2007). In the present study, we quantitatively investigated the white matter and the myelinated fibers of the white matter in the rat brain and compared this data with the human brain. As shown in Table 2, the volume of human white matter was 3958 times larger than that of rat white matter. However, after analyzing the volume density and length density of the myelinated fibers in the white matter, we found that in rat white matter, the myelinated fibers were densely packed when compared to the myelinated fibers in human white matter. The volume density of the myelinated fibers in rat white matter was $48.5 \%$ larger than that in human white matter (Table 2). Surprisingly, the length density of the myelinated fibers in rat white matter was $332 \%$ larger than that in human white matter (Table 2). In contrast, the mean diameter of the myelinated fibers in rat white matter was $51 \%$ smaller than that in human white matter (Table 2). The results suggested that the myelinated fibers in rat white matter were proportionally much smaller than the myelinated fibers in human white matter. The structural differences between rat white matter and human white matter will provide valuable data for the future studies on the functional differences between rat brain and human brain. The average diameter of $0.56 \mu \mathrm{m}$ for the myelinated fibers of the Long-Evans rat in the current study is similar to an average diameter of $0.44-0.60 \mu \mathrm{m}$ for the myelinated fibers of various subcortical white matter regions in the Wistar rat (Partadiredja et al., 2003).

For the main outcome measures, the biological variation makes up approximately $80 \%$ of the observed variance. The results were satisfactory in the sense that the sampling variance introduced by the stereological estimation procedure was a minor fraction of the observed variance. Therefore, it was not necessary to count more nerve fibers and not necessary to analyze the used sampling scheme any further.

In conclusion, this study presents for all practical purposes unbiased stereological sampling techniques for estimating the total volume and total length of the myelinated fibers in the white matter, the total volume of the myelin sheaths in the white matter and the mean diameter of the myelinated fibers in the white matter of Long-Evans rat.

\section{ACKNOWLEDGEMENTS}

This study was supported by National Natural Science Foundation (NSF 30440082, 30572075), 2005 Financial Support for the Young Talented Scientists Returning to China from the Ministry of Personnel, Key Projects from the Ministry of Education, Research Start Foundation for the Persons Returning to China from the Ministry of Education, P. R. China, Natural Science Foundation of Chongqing Government (SCTC, 2005BB5034), Research Projects from the Committee of Education, Chongqing Government. We thank all the staff in the Department of the Electron Microscope, Chongqing Medical University, P. R. China for their assistance in the TEM technique. We gratefully acknowledge the Stereology and Electron Microscopy Research Laboratory and MIND Center, Aarhus University, Denmark for providing the isector moulds.

\section{REFERENCES}

Braendgaard H, Gundersen HJG (1986). The impact of recent stereological advances on quantitative studies of the nervous system. J Neurosci Meth 18:39-78.

Christiansen P, Larsson HB, Thomsen C, Wieslander SB, Henriksen O (1994). Age dependent white matter lesions and brain volume changes in healthy volunteers. Acta Radiol 35:117-22.

de Wit S, Niry D, Wariyar R, Aitken MR, Dickinson A (2007). Stimulus-outcome interactions during instrumental discrimination learning by rats and humans. J Exp Psychol Anim Behav Process 33:1-11.

Erickson CA, Barnes CA (2003). The neurobiology of memory changes in normal aging. Exp Gerontol 38:61-9.

Ge Y, Grossman RI, Babb JS, Rabin ML, Mannon LJ, Kolson DL (2002). Age-related total gray matter and white matter changes in normal adult brain. Part (1): volumetric MR imaging analysis. ANJR Am J Neuroradiol 23:1327-33.

Gundersen HJ, Bendtsen TF, Korbo L et al. (1988). Some new, simple and efficient stereological methods and their use in pathological research and diagnosis. AMPIS 96:379-94.

Gundersen HJ, Jensen EB, Kiêu K, Nielsen J (1999). The efficiency of systematic sampling in stereology-reconsidered. J Microsc 193:199-211.

Gundersen HJ (1977). Notes on the estimation of the numerical density of arbitrary particles: The edge effect. J Microsc 111:219-23. 
Gundersen HJ (1979). Estimation of tubule or cylinder Lv, Sv, and Vv on thick sections. J Microsc 117:333-45.

Guttmann CR, Jolesz FA, Kikinis R et al. (1998). White matter changes with normal aging. Neurology 50:972-8.

Kroustrup JP, Gundersen HJ (1983). Sampling problems in an heterogeneous organ: quantitation of relative and total volume of pancreatic islets by light microscopy. J Microsc 132:43-55.

Lammers JH, Emmen HH, Muijser H et al. (2007). Model studies for evaluating the neurobehavioral effects of complex hydrocarbon solvents II. Neurobehavioral effects of white spirit in rat and human. Neurotoxicology 28:736-50.

Malloy P, Correia S, Stebbins G, Laidlaw DH (2007). Neuroimaging of white matter in aging and dementia. Clin Neuropsychol 21:73-109.

Marner L, Nyengaard JR, Tang Y, Pakkenberg B (2003). Marked loss of myelinated fibers in human brain with age. J Comp Neurol 462:144-52.

Meier-Ruge W, Ulrich J, Brühlmann M, Meier E (1992). Age-related white matter atrophy in the human brain. Ann N Y Acad Sci 673:260-9.

Nyengaard JR, Gundersen HJ (1992). The isector: a simple and direct method for generating isotropic, uniform random sections from small specimens. J Microsc 165: 427-31.

Pang K, Merkel F, Egeth H, Olton DS (1992). Expectancy and stimulus frequency: a comparative analysis in rats and humans. Percept Psychophys 51:607-15.

Partadiredja G, Miller R, Oorschot DE (2003). The number, size, and type of axons in rat subcortical white matter on left and right sides: a stereological, ultrastructural study. J Neurocytol 32:1165-79.

Resnick SM, Pham DL, Kraut MA, Zonderman AB, Davatzikos C (2003). Longitudinal magnetic resonance imaging studies of older adults: a shrinking brain. $\mathrm{J}$ Neurosci 23:3295-301

Sargon MF, Mas N, Senan S, Ozdemir B, Celik HH, Cumhur M (2003). Quantitative analysis of myelinated axons of commissural fibers in the rat brain. Anat Histol Embryol 32:141-4.
Shu SY, Wu YM, Bao XM et al. (2002). A new area in the human brain associated with learning and memory: immunohistochemical and functional MRI analysis. Molecular Psychiatry 7:1018-22.

Sturrock RR (1976). Changes in neuroglia and myelination in the white matter of aging mice. J Gerontol 31:513-22.

Sturrock RR (1987). Age-related changes in the number of myelinated axons and glial cells in the anterior and posterior limbs of the mouse anterior commissure. $\mathrm{J}$ Anat 150:111-27.

Tang Y, Nyengaard JR (1997). A stereological method for estimating the total length and size of myelin fibers in human brain white matter. J Neurosci Meth 73:193-200.

Tang Y, Nyengaard JR (2004). Length estimation of nerve fibers in human white matter using IUR sections. In: Evans SM, Janson AM, Nyengaard JR, eds. Quantitative Methods in Neuroscience-a Neuroanatomical Approach. Oxford University Press Inc., New York, 249-63.

Tang Y, Nyengaard JR, Pakkenberg B, Gundersen HJ (1997). Age-induced white matter changes in the human brain: A stereological investigation. Neurobiol Aging 18:609-15.

Tang Y, Nyengaard JR, Pakkenberg B, Gundersen HJ (2003). Stereology of neuronal connections (myelinated fibers of white matter and synapses of neocortex) in human brain. Image Anal Stereol 20:171-82.

Wallace DG, Choudhry S, Martin MM (2006). Comparative analysis of movement characteristics during deadreckoning-based navigation in humans and rats. J Comp Psychol 120:331-44.

West MJ, Gundersen HJ (1990). Unbiased stereological estimation of the number of neurons in the human hippocampus. J Comp Neurol 296:1-22.

West MJ, Slomianka L, Gundersen HJ (1991). Unbiased stereological estimation of the total number of neurons in the subdivisions of the rat hippocampus using the optical fractionator. Anat Rec 231:482-97.

Ylikoski A, Erkinjuntti T, Raininko R, Sarna S, Sulkava R, Tilvis R (1995). White matter hyperintensities on MRI in the neurologically nondiseased elderly. Analysis of cohorts of consecutive subjects aged 55 to 85 years living at home. Stroke 26:1171-7. 